3. Godfrey JD, Brantner TL, Brinjikji W, et al. Morbidity and mortality among older individuals with undiagnosed coeliac disease. Gastroenterology 2010;139:763-9.

4. Long KH, Rubio-Tapia A, Wagie AE, et al. The economics of celiac disease: a population-based study. Aliment Pharmacol Ther 2010:32:261-9.

\section{PTU-138 ANALYSIS OF THE EFFECTS OF SPECIALISATION ON THE QUALITY OF REPORTING OF STOMACH CANCER USING THE ROYAL COLLEGE OF PATHOLOGISTS MINIMUM DATASET IN THE YORKSHIRE REGION}

doi:10.1136/gutjnl-2012-302514c.138

${ }^{1} 0$ Rotimi, ${ }^{*}{ }^{2}$ E Morris. ${ }^{1}$ Histopathology, Leeds Teaching Hospitals Trust, Leeds, UK; ${ }^{2}$ NYCRIS, Leeds Teaching Hospitals Trust, Leeds, UK

Introduction The completeness of a cancer pathology report is central to quality patient management and over the years proforma reporting has become a way of standardising reports. There has been an increasing trend towards specialisation in histopathology. This study examines the effect of specialisation on the degree of completeness, accuracy of information and quality of reporting of the Royal College of Pathologists' stomach cancer dataset.

Methods An audit of 1065 pathology forms from surgically removed stomach cancer cases over a 12-year period (1995-2006) was carried out. The rate of completeness of the forms, accuracy of the information content and quality of reporting were determined. Accuracy of the information content was adjudged by running specific queries to check for discrepancies such as mis-match of the depth of local invasion and the pathological tumour (pT) stage. Quality was assessed by the number of lymph nodes retrieved and recorded. The impact of specialisation on the accuracy and quality of the information contents of the forms were analysed by comparing median number of lymph nodes retrieved, completion rate of the forms and rate of discrepancies between specialist (Leeds) and non-specialist (11 others) departments. Differences between the two were statistically tested for significance $(p \leq 0.05)$ using appropriate parametric and non-parametric tests.

Results Of the 1065 forms, 31\% were submitted from Leeds NHS Trust. There was $90.3 \%$ completion rate for core items overall and there were discrepancies in 316 cases $(30 \%)$. The number of lymph nodes retrieved range from 0 to 95 per case with an overall median of 15 lymph nodes. The results of the comparison between the specialist and non-specialist centres with respect to completeness, accuracy and quality are presented in Abstract PTU-138 table 1. These showed statistically significant difference between the two groups with specialist centre consistently performing better than the non-specialist centres.

Abstract PTU-138 Table 1 Assessing role of specialisation on quality

\begin{tabular}{lrcr}
\hline Variable & Specialist & Non-specialist & p Value \\
\hline $\begin{array}{l}\text { Lymph nodes retrieved } \\
\text { Median (IOR) }\end{array}$ & $22(14-30)$ & $14(8-19)$ & $0.0001^{*}$ \\
$\begin{array}{l}\text { Completion rate of forms } \\
\quad \text { Complete }(\mathrm{n}=858)\end{array}$ & $281(85.7 \%)$ & $571(78.2 \%)$ & \\
$\quad$ Incomplete $(\mathrm{n}=207)$ & $48(14.3 \%)$ & $159(21.8 \%)$ & $0.004 \dagger$ \\
Discrepancies on forms $(\mathrm{n}=316)$ & $81(25.6 \%)$ & $235(74.4 \%)$ & $<0.0001 \dagger$ \\
\hline
\end{tabular}

*Kruskal-Wallis rank test.

$+\chi^{2}$ test.

Conclusion This analysis of a large number of proformas from a region shows a completion rate and an overall good quality using lymph node retrieval. Specialisation of histopathologists has significant impact on the completeness of forms, accuracy of information content and quality of reporting. Therefore, further specialisation is recommended to improve the quality of cancer reports and patient management.

Competing interests None declared.

\section{PTU-139 COLONIC HISTOLOGICAL ABNORMALITIES ARE NON- SPECIFIC AND NON-SIGNIFICANT IN BILE ACID MALABSORPTION}

doi:10.1136/gutjnl-2012-302514c.139

${ }^{1} 0$ Orekoya,* ${ }^{2} \mathrm{~J}$ McLaughlin, ${ }^{3} \mathrm{E}$ Leitao, ${ }^{3} \mathrm{~W}$ Johns, ${ }^{2} \mathrm{P}$ Paine. ${ }^{1}$ University of Manchester, Manchester, UK; ${ }^{2}$ Gastroenterology, Salford Royal Foundation Trust, Manchester, UK; ${ }^{3}$ Nuclear Medicine, Salford Royal Foundation Trust, Manchester, UK

Introduction Bile acid malabsorption (BAM) is a common cause of chronic secretory diarrhoea via poorly characterised mechanisms. The aim of this study was to determine if histological abnormalities in colonic mucosa are linked to BAM in patients with chronic diarrhoea.

Methods During a 6-year period 264 patients were investigated with SeHCAT for chronic diarrhoea and their retention values recorded $(\leq 8 \%=$ positive result; $\geq 16 \%=$ negative result). Colonic biopsies and histological analysis were available in 150 (57\%). The patients were categorised as: Group 1: terminal ileum Crohn's disease, (pre or post resection) $\mathrm{n}=51$. Group 2: Idiopathic BAM (including patients with diarrhoea predominant IBS) $n=159$. Group 3: BAM secondary to other gastrointestinal disease $n=51$; of which cholecystectomy $(n=37)$, coeliac disease $(n=1)$, chronic pancreatitis $(n=1)$, bacterial overgrowth $(n=2)$, diabetes $(n=4)$ and other gastrointestinal surgeries $(n=6)$. Group 4: terminal ileum disease plus cholecystectomy $\mathrm{n}=3$.

Results A histological abnormality was present in $29 \%(n=16 / 56)$ of the patients with positive SeHCAT, vs $23 \%(n=17 / 74)$ of the patients with negative SeHCAT ( $p=0.318$, two-tailed Fisher's exact test). Similarly, $30 \%(n=6 / 20)$ of the patients with equivocal SeHCAT results ( $8 \%-16 \%$ retention) had histological abnormalities. As expected, the highest prevalence of these abnormalities was noted in groups 1 and 4, the subjects with Crohn's disease, regardless of the presence of BAM. Abnormalities were noted in descriptive terms and included colitis $(n=4)$, chronic inflammation $(n=5)$, cryptitis $(n=4)$, mild non-specific inflammation $(n=11)$, moderate non-specific inflammation $(n=5)$, polyps $(n=2)$, ulceration $(n=6)$ and active inflammatory bowel disease $(n=2)$. $30 \%(n=8 / 27)$ of positive SeHCAT with idiopathic BAM (group 2) had abnormalities on histology compared with $19 \%(n=10 / 52)$ who had negative SeHCAT in group 2 , which was again a non-significant difference $(p=0.397$, two-tailed Fisher's exact test). The $7 \%(n=2 / 28)$ of patient in group 3 with histological abnormalities also had negative SeHCAT results. Conclusion This retrospective study indicates that colonic histological abnormality is non-specific and coincidental to the presence of bile acid malabsorption in patients with chronic diarrhoea. It is unlikely to be relevant to the mechanism of diarrhoea which is probably non-inflammatory. This might be further studied in the future using faecal markers of inflammation in these patients.

Competing interests None declared.

\section{PTU-140 NEUTROPHIL LYMPHOCYTE RATIO IN OUTCOME PREDICTION AFTER EMERGENCY ABDOMINAL SURGERY IN THE ELDERLY}

doi:10.1136/gutjnl-2012-302514c.140

${ }^{1} \mathrm{P}$ G Vaughan-Shaw, ${ }^{2}$ 'J R Rees, ${ }^{1}$ A T King. 'Department of Lower Gl Surgery, University Hospitals Southampton Foundation Trust, Southampton, UK; ${ }^{2}$ Academic Unit of Surgical Research, Section of Healthcare Research, University of Bristol, Bristol, UK

Introduction Accurate prediction of outcome after emergency surgery in the elderly patient may assist decision making. However certain scoring systems require post-operative data (eg, P-POSSUM) while others have failed to gain widespread use (eg, Surgical risk score). Recent reports have suggested that $\mathrm{C}$ reactive protein (CRP) 